\title{
Clues to NaCN formation ${ }^{\star}$
}

\author{
G. Quintana-Lacaci ${ }^{1}$, J. Cernicharo ${ }^{1}$, L. Velilla Prieto ${ }^{1}$, M. Agúndez ${ }^{1}$, A. Castro-Carrizo ${ }^{2}$, J. P. Fonfría ${ }^{1}$, \\ S. Massalkhi ${ }^{1}$, and J. R. Pardo ${ }^{1}$ \\ 1 Instituto de Ciencia de Materiales de Madrid, Sor Juana Inés de la Cruz, 3, Cantoblanco, 28049 Madrid, Spain \\ e-mail: g.quintana@icmm.csic.es \\ 2 Institut de RadioAstronomie Millimétrique, 300 rue de la Piscine, 38406 Saint-Martin d'Hères, France
}

Received 28 September 2017 / Accepted 20 October 2017

\begin{abstract}
Context. ALMA is providing us essential information on where certain molecules form. Observing where these molecules emission arises from, the physical conditions of the gas, and how this relates with the presence of other species allows us to understand the formation of many species, and to significantly improve our knowledge of the chemistry that occurs in the space.

Aims. We studied the molecular distribution of $\mathrm{NaCN}$ around $\mathrm{IRC}+10216$, a molecule detected previously, but whose origin is not clear. High angular resolution maps allow us to model the abundance distribution of this molecule and check suggested formation paths.

Methods. We modeled the emission of $\mathrm{NaCN}$ assuming local thermal equilibrium (LTE) conditions. These profiles were fitted to azimuthal averaged intensity profiles to obtain an abundance distribution of $\mathrm{NaCN}$.

Results. We found that the presence of $\mathrm{NaCN}$ seems compatible with the presence of $\mathrm{CN}$, probably as a result of the photodissociation of $\mathrm{HCN}$, in the inner layers of the ejecta of IRC +10216 . However, similar as for $\mathrm{CH}_{3} \mathrm{CN}$, current photochemical models fail to reproduce this $\mathrm{CN}$ reservoir. We also found that the abundance peak of $\mathrm{NaCN}$ appears at a radius of $3 \times 10^{15} \mathrm{~cm}$, approximately where the abundance of $\mathrm{NaCl}$, suggested to be the parent species, starts to decay. However, the abundance ratio shows that the $\mathrm{NaCl}$ abundance is lower than that obtained for $\mathrm{NaCN}$. We expect that the LTE assumption might result in $\mathrm{NaCN}$ abundances higher than the real ones. Updated photochemical models, collisional rates, and reaction rates are essential to determine the possible paths of the $\mathrm{NaCN}$ formation.
\end{abstract}

Key words. astrochemistry - stars: AGB and post-AGB - circumstellar matter - stars: individual: IRC+10216

\section{Introduction}

During its few cycles of operation, the ALMA interferometer has proved to be an incomparable tool for the study of molecule formation in space. A particular well-suited source for such studies is the C-rich AGB star IRC +10216 , one of the closest evolved stars, located at a distance of $\sim 123 \mathrm{pc}$ from us (Groenewegen et al. 2012). While this object has been intensively studied, in particular in the field of the astrochemistry (see, e.g., Cernicharo et al. 2000, 2013; Agúndez et al. 2012, etc.), essential information on the exact location of the different molecular reservoirs has remained inaccessible for years because of the limited angular resolution of the instruments operating at (sub-)millimeter (mm) wavelengths.

Only recently has the scientific exploitation of ALMA allowed us to understand with unprecedented detail the structure and kinematics of the circumstellar envelope of IRC +10216 (Cernicharo et al. 2015; Decin et al. 2015; Quintana-Lacaci et al. 2016; Guélin et al. 2017) as well as the exact regions where the

\footnotetext{
* Based on observations carried out with ALMA and the IRAM $30 \mathrm{~m}$ Telescope. ALMA is a partnership of ESO (representing its member states), NSF (USA) and NINS (Japan), together with NRC (Canada) and NSC and ASIAA (Taiwan), in cooperation with the Republic of Chile. The Joint ALMA Observatory is operated by ESO, AUI/NRAO and NAOJ. IRAM is supported by INSU/CNRS (France), MPG (Germany) and IGN (Spain). This paper makes use of the following ALMA data: ADS/JAO.ALMA\#2013.1.00432.S \& ADS/JAO.ALMA\#2016.1.01217.S.
}

different species are formed (Agúndez et al. 2015, 2017; Velilla Prieto et al. 2015).

The location of the different molecules in the ejecta is crucial to understand the chemical processes that are at work in IRC +10216 , in particular, and in circumstellar envelopes in general. In this work we study the distribution of the metal-bearing molecule $\mathrm{NaCN}$ around IRC +10216 and explore its probable origin and the limitations of current chemical models. This work highlights once again the deep importance of the synergy between high-spatial resolution observations and laboratory and theoretical work to obtain collisional rates and chemical reaction rate constants.

\section{Observations and $\mathrm{NaCN}$ distribution}

We carried out a $\lambda 3 \mathrm{~mm}$ spectral survey of IRC +10216 with ALMA band 3 during Cycle 2, covering the frequency range 84.0-115.5 GHz. In addition, we recently obtained a mosaic with ALMA covering selected frequencies at $2 \mathrm{~mm}$. These observation are described in detail in Appendix A.

We obtained interferometric maps of the $\mathrm{NaCN}$ transitions presented in Table 1, both merging ALMA compact configuration data and the on-the-fly (OTF) maps obtained with the IRAM $30 \mathrm{~m}$ telescope, and the ALMA data in the compact and extended configurations with the OTF single-dish maps for the observations of Cycle 2. This resulted in two sets of maps, one with a typical angular resolution of $4^{\prime \prime} \times 3^{\prime \prime}$, and another with a higher angular resolution of $0 .{ }^{\prime \prime} 8 \times 0 . \prime 7$. 
Table 1. Parameters of the observed lines.

\begin{tabular}{|c|c|c|c|c|c|c|c|c|}
\hline \multirow[b]{2}{*}{ Molecule } & \multirow[b]{2}{*}{ Trans. } & \multirow[b]{2}{*}{ Freq $(\mathrm{MHz})$} & \multirow[b]{2}{*}{$E_{\text {up }}(\mathrm{K})$} & \multirow[b]{2}{*}{$S_{i j}$} & \multicolumn{2}{|c|}{ Low spatial resolution } & \multicolumn{2}{|c|}{ High spatial resolution } \\
\hline & & & & & Beam & $\mathrm{PA}\left({ }^{\circ}\right)$ & Beam & $\mathrm{PA}\left({ }^{\circ}\right)$ \\
\hline $\mathrm{NaCN}$ & $6_{1,6}-5_{1,5}$ & 90394.38 & 17.6 & 5.83237 & $3 .{ }^{\prime \prime} 9 \times 2 . .7$ & 89.9 & $0 .{ }^{\prime \prime} 8 \times 0.0^{\prime \prime} 6$ & 39.2 \\
\hline $\mathrm{NaCN}$ & $60,6-5_{0,5}$ & 93206.09 & 15.7 & 5.99388 & $4 . .3 \times 2 . .7$ & 72.1 & $0 .^{\prime \prime} 6 \times 0.0^{\prime \prime} 6$ & 15.2 \\
\hline $\mathrm{NaCN}$ & $6_{2,4}-5_{2,3}$ & 94334.80 & 25.4 & 5.33293 & $4 .{ }^{\prime \prime} 3 \times 2.6$ & 72.4 & $0 .^{\prime \prime} 6 \times 0.0^{\prime \prime} 6$ & 40.4 \\
\hline $\mathrm{NaCN}$ & $6_{1,5}-5_{1,4}$ & 96959.81 & 18.7 & 5.83223 & 3." $9 \times 2 . .6$ & 92.7 & $0 . \prime 8 \times 0 . \prime 7$ & 20.0 \\
\hline $\mathrm{NaCN}$ & $9_{1,8}-8_{1,7}$ & 145075.57 & 37.3 & 8.88452 & $1{ }^{\prime \prime} 4 \times 11^{\prime \prime} 1^{*}$ & 52.4 & & \\
\hline $\mathrm{NaCN}$ & $10_{2,8}-9_{2,7}$ & 158616.77 & 51.2 & 9.59903 & $1{ }^{\prime \prime} 3 \times 1{ }^{\prime \prime} 0^{*}$ & 65.1 & & \\
\hline $\mathrm{NaCl}$ & $7-6$ & 91169.88261 & 17.5 & 7.0 & - & - & $0 .{ }^{\prime \prime} 8 \times 0.0^{\prime \prime} 6$ & 38.8 \\
\hline $\mathrm{CH}_{3} \mathrm{CN}$ & $66_{3}-5_{3}$ & 110364.35314 & 82.8 & 9.0 & - & - & $0 . \prime 8 \times 0.7$ & 39.1 \\
\hline $\mathrm{CH}_{3} \mathrm{CN}$ & $6_{0}-5_{0}$ & 110383.49871 & 18.5 & 6.0 & - & - & $0 .{ }^{\prime \prime} 8 \times 0.7$ & 38.9 \\
\hline
\end{tabular}

Notes. High spatial resolution: short-spacing and ALMA compact data merged. Low spatial resolution: short-spacing and ALMA compact and extended data merged. ${ }^{(*)}$ Only ALMA visibilities.

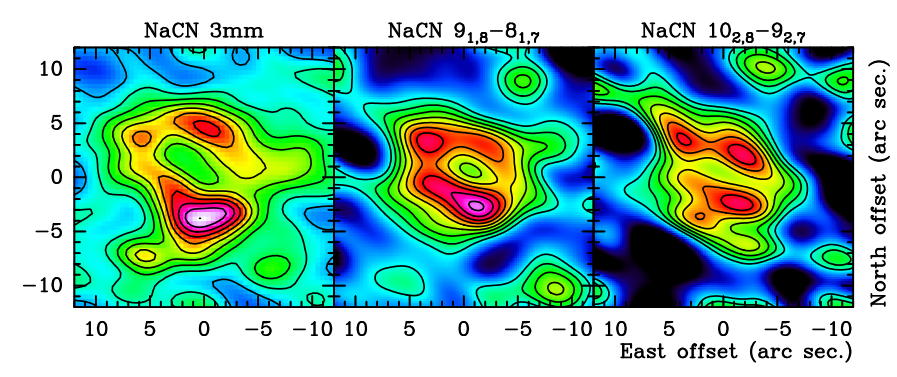

Fig. 1. Comparison of the central channel of the low spatial resolution $3 \mathrm{~mm}$ stacked map with maps of the ALMA Cycle 4 observations at $2 \mathrm{~mm}$. The lowest contour corresponds to $10 \%$ of the peak flux, and the rest of contours are equally spaced in jumps of $10 \%$ with respect to the first contour. For absolute intensities see Figs. A.1, A.4, and A.5.

The sensitivity of the individual maps of each transition is not high enough to have a clear view of the distribution of the $\mathrm{NaCN}$ emitting gas. The brightness distribution that is visible in the velocity channels suggests a spherical hollow shell-like distribution. In order to increase the signal-to-noise ratio $(\mathrm{S} / \mathrm{N})$ and to confirm this brightness distribution, we stacked the emission from the $\mathrm{NaCN}$ lines presented in Table 1. In the case of the low angular resolution maps, this stacking already allowed us to confirm the hollow shell gas distribution suggested by the single maps (see Fig. A.1). However, in the case of the high spatial resolution maps, the stacking alone was not enough. We therefore smoothed the spectral resolution to $5 \mathrm{~km} \mathrm{~s}^{-1}$ to increase the $\mathrm{S} / \mathrm{N}$. This high spatial - low spectral resolution map is presented in Fig. A.2.

In addition, we merged the visibilities of the seven fields for Cycle $4 \mathrm{NaCN}$ data and obtained cleaned maps with an angular $1^{\prime \prime}$. These maps presented negative fluxes and a smaller extent than the $3 \mathrm{~mm} \mathrm{NaCN}$ data, confirming the flux loss. Similar as for the $3 \mathrm{~mm}$ data, we reduced the spatial resolution to increase the $\mathrm{S} / \mathrm{N}$. In particular, we imposed in the cleaning algorithm the synthezised beam to obtain the same spatial resolution as in the map presented in Fig. A.1. These maps are presented in Figs. A.4 and A.5. The NaCN brightness distribution of the stacked map of the $3 \mathrm{~mm}$ lines and from the two $\mathrm{NaCN}$ transitions observed at $2 \mathrm{~mm}$ show the very same structure, confirming the presence of the hollow shell mentioned above (Fig. 1).

\section{NaCN modeling}

In order to fit the observed gas distribution, we assumed the density and temperature profiles adopted by Quintana-Lacaci et al. (2016), slightly modified to follow the temperature law deduced by Guélin et al. (2017). Similarly to what was done in that work, the abundance profile was adjusted to the observation using as starting point the abundance profiles derived by Agúndez et al. (2012). We only aimed at fitting the $3 \mathrm{~mm}$ data because for these transitions all the flux has been recovered by merging the ALMA visibilities with the short-spacing data.

Since there are no available collisional rates for $\mathrm{NaCN}$, we used a local thermodynamical equilibrium (LTE) multi-shell approach carried out with the MADEX code (Cernicharo 2012) to reproduce the observations. In this approach, we solve the level populations at the different radii, assuming LTE conditions. When this is solved, the synthetic profiles are obtained by solving the ray tracing and convolving with the beam of the telescope.

As for fitting the abundance profile, we assumed that the emission distribution is mainly spherical. Therefore, we obtained the azimuthal averaged emission of the $\mathrm{NaCN}$ stacked map for both the high- and the low-resolution maps. We aimed at fitting both emission profiles at the same time, taking into account that the $\mathrm{S} / \mathrm{N}$ difference of the low-resolution and high-resolution maps is significant. A good fitting of the high $\mathrm{S} / \mathrm{N}$ emission profile was mandatory, while a reasonable fit of the low $\mathrm{S} / \mathrm{N}$ profile was enough. These fits are shown in Fig. 3.

\section{Discussion}

The location of the different metal-bearing cyanides can be separated into two main groups. Some of them, such as MgNC (Guélin et al. 1993) and HMgNC (Cabezas et al. 2013), are found to appear far from the star's photosphere, forming a shelllike distribution with a radius of $\sim 15^{\prime \prime}$. This structure has been confirmed in MgNC by our present ALMA observations (see Fig.A.3). In contrast, species such as $\mathrm{NaCN}$ were found to present compact emission when observed with a spatial resolution of $3^{\prime \prime}$ (Guélin et al. 1993, 1997). These latter results suggested that the formation of $\mathrm{NaCN}$ takes place in chemical equilibrium conditions in the vicinity of the stellar photosphere. However, the maps we present here reveal a small inner hole with a radius $\sim 1.5^{\prime \prime}$ in the brightness distribution of $\mathrm{NaCN}$, suggesting that it is formed in regions where the gas has left the chemical equilibrium regime.

These two-fold distribution of the metal cyanides has been theoretically explained by Petrie (1996) based on the physical characteristics of the parent species that combine with $\mathrm{CN}$. In particular, metal-bearing species such as $\mathrm{AlCl}, \mathrm{NaCl}$, or $\mathrm{KCl}$ are 


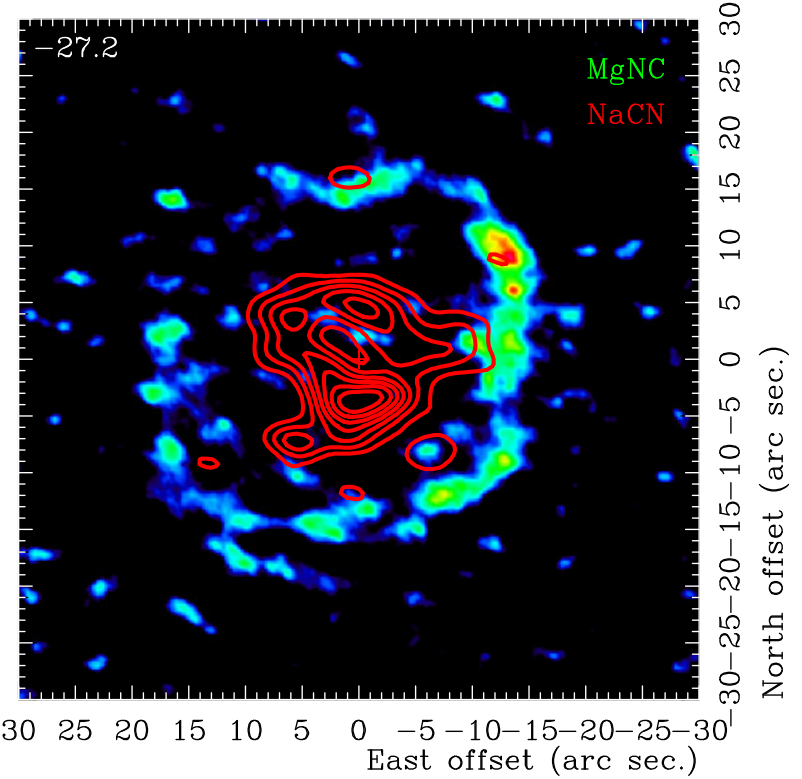

Fig. 2. Comparison of the extent of emission between $\mathrm{NaCN}$ (red contours, see Fig. A.1) and MgNC (color scale, see Fig. A.3).

closed-shell molecules that form in the inner hot regions of the envelope, while other species containing $\mathrm{Mg}, \mathrm{Fe}$, or $\mathrm{Si}$ are openshell radicals that could react with other neutral molecules in regions with low temperatures. Reactions involving $\mathrm{CN}$ and the former group of species would therefore result in metal cyanides in the innermost regions of the ejecta, while those involving the latter group will result in cyanides forming extended shell-like structures like those cited above. A comparison of the extent of these diferent cyanides is shown in Fig. 2.

In the particular case of $\mathrm{NaCN}$, Petrie (1996) suggested the following pathway:

$\mathrm{NaCl}+\mathrm{CN} \rightarrow \mathrm{NaCN}+\mathrm{Cl}$.

Since $\mathrm{NaCl}$ is a closed-shell species that is abundant in the innermost regions of the envelope (Quintana-Lacaci et al. 2016), this reaction could take place when a significant amount of $\mathrm{CN}$ is available. The distribution of the $\mathrm{NaCl} J=7-6$ emission, with an energy of the upper level $\left(E_{\text {up }}\right)$ similar to the $\mathrm{NaCN}$ transitions we analyzed here and obtained within the same ALMA Cycle 2 project (see Appendix A and Table 1 for details), showed that its brightness distribution is complementary to that of $\mathrm{NaCN}$, that is, when $\mathrm{NaCl}$ emission fades, $\mathrm{NaCN}$ emission rises. This is shown in Fig. 4 by comparing the azimuth-averaged emission of the central velocity channel for the different transitions. This seems to support the assumption that $\mathrm{NaCl}$ is a parent molecule for the formation of $\mathrm{NaCN}$.

On the other hand, the models suggest that $\mathrm{CN}$ is present only near the photosphere and in the outer layers of the CSE as a result of the photodissociation of HCN (Lucas et al. 1995). Recent ALMA CN maps confirm that the CN emission appears at typical radii of $\sim 15^{\prime \prime}$ (Agúndez et al. 2017).

Recently, Agúndez et al. (2015) showed that the $\mathrm{CH}_{3} \mathrm{CN}$ spatial distribution was unexpectedly located in an inner hollow shell. To study the possible relation of the formation of $\mathrm{NaCN}$ with that of $\mathrm{CH}_{3} \mathrm{CN}$, we have compared the brightness distribution of this molecule with that of the stacked $\mathrm{NaCN}$ map. While $\mathrm{CH}_{3} \mathrm{CN} 6_{0}-5_{0}$ has a excitation temperature similar to that of the $\mathrm{NaCN}$ transitions we present here, it is blended with the $6_{1}-5_{1}$. We therefore used the unblended transition $6_{3}-5_{3}$ for the comparison. The two azimuth-averaged maps present very similar

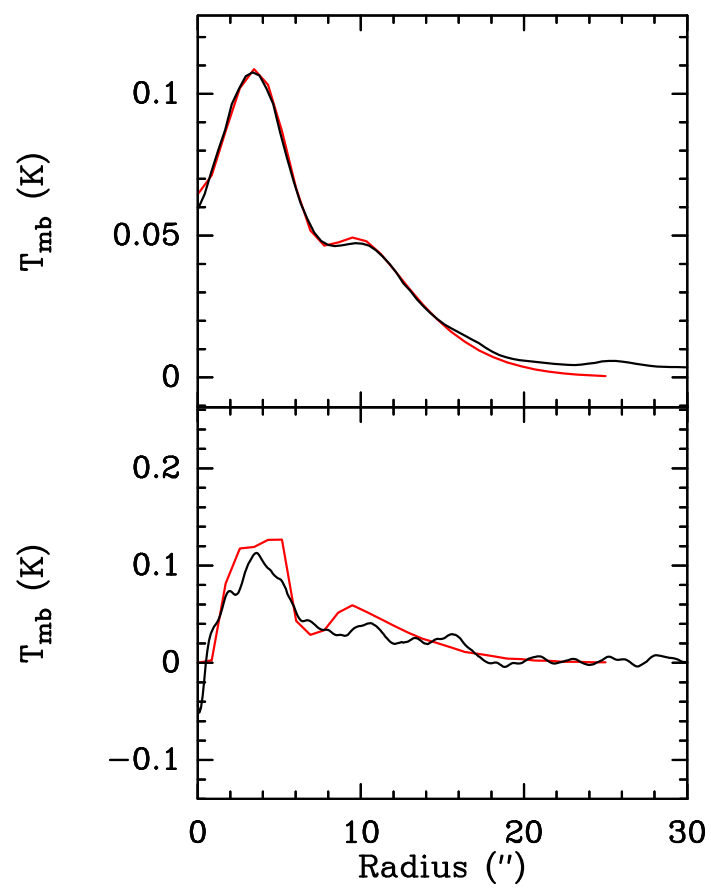

Fig. 3. Top: model fitting (red line) of the azimuthally averaged emission of the stacked $\mathrm{NaCN}$ lines presented in Table 1 for the low spatial resolution maps (Fig. A.1). Bottom: model fitting (red line) of the azimuthally averaged emission of the stacked $\mathrm{NaCN}$ lines presented in Table 1 for the high spatial resolution maps (Fig. A.2).

distributions, suggesting that the parent molecule responsible for both molecules might be similar. In particular, an injection of $\mathrm{CN}$ at a radii of $\sim 1^{\prime \prime} .5-2^{\prime \prime}$ could explain the distribution of both $\mathrm{CH}_{3} \mathrm{CN}$ and $\mathrm{NaCN}$. Such an injection would also affect other species, in particular, $\mathrm{HC}_{3} \mathrm{~N}$. However, since the rate constant of reaction (1) is unknown, we cannot estimate the balance between the different reactions involving $\mathrm{CN}$.

Furthermore, Agúndez et al. (2015) showed that the model presented by Agúndez et al. (2010), which takes into account the effect of penetrating UV photons in the innermost layers of the circumstellar envelope (CSE) around IRC +10216 fails to explain the $\mathrm{CH}_{3} \mathrm{CN}$ distribution. Therefore, to understand the $\mathrm{CN}$ source and reaction distribution, a detailed new model is mandatory. This model will be developed in a forthcoming paper.

The results shown in Fig. 4 clearly suggest that the reaction proposed by Petrie (1996) is plausible and that a certain amount of cyanide is freed by photodissociation of $\mathrm{HCN}$ or other $\mathrm{CN}-$ bearing species with weaker bounds than NCCN, not detected in space so far, and that it reacts rapidly to form $\mathrm{NaCN}$ and $\mathrm{CH}_{3} \mathrm{CN}$.

\section{Results}

The abundance profile obtained for $\mathrm{NaCN}$ is presented in Fig. 5 . As suggested by the $\mathrm{NaCN}$ emission maps (see Figs. A.1 and A.2), we found that $\mathrm{NaCN}$ arises at a radius of $3 \times 10^{15} \mathrm{~cm}$.

To check the abundance relation between $\mathrm{NaCN}$ and $\mathrm{NaCl}$, we compared the abundance profile obtained here with that derived by Quintana-Lacaci et al. (2016) for $\mathrm{NaCl}$ (dashed line in Fig. 5). This comparison shows that the tentative precursor has a lower abundance than the resulting species. This might indicate that reaction (1) is not the main formation path of $\mathrm{NaCN}$. However, we have to keep in mind that while the $\mathrm{NaCl}$ abundance profile was accurately derived by solving the level 


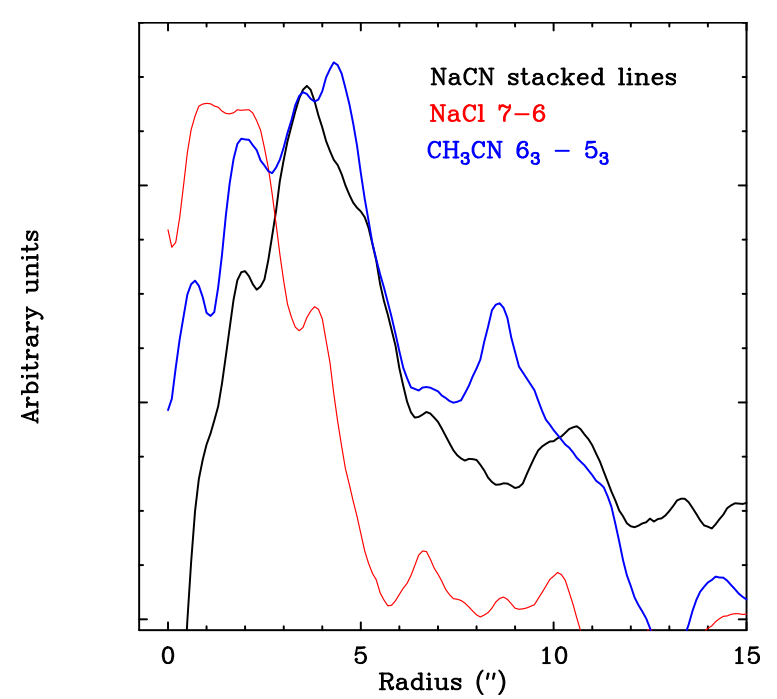

Fig. 4. Comparison of the azimuthally averaged intensity profiles of the $\mathrm{NaCN}$ maps (black line), the $\mathrm{NaCl} 7-6$ emission map (red line), and the $\mathrm{CH}_{3} \mathrm{CN} 6_{3}-5_{3}$ (blue line).

population in non-LTE conditions, that of $\mathrm{NaCN}$ has been derived assuming LTE.

At the regions where $\mathrm{NaCN}$ abundance rises $\left(3 \times 10^{15} \mathrm{~cm}\right)$ $T_{\mathrm{K}} \sim 160 \mathrm{~K}$ (Guélin et al. 2017). This means that because LTE assumes $T_{\text {ex }}=T_{\mathrm{K}}$, the high-excitation lines are favored over low-excitation lines such as we studied here. The regions where, in LTE, these low-excitation transitions are expected to dominate lie at radii $\sim 2.5 \times 10^{16} \mathrm{~cm}$. However, as shown by Agúndez et al. (2012), metal-bearing species studied by these authors leave the LTE regime at the regions where $\mathrm{NaCN}$ emission arises. Furthermore, these authors showed that the line intensity ratios from the $\mathrm{NaCN}$ lines observed are not compatible with an LTE regime. Therefore, we might expect non-LTE modeling to derive lower values of $T_{\mathrm{ex}}$ and therefore higher level populations, higher intensities, and lower abundances for the transitions observed. This lower abundance would then conciliate the $\mathrm{NaCl}$ and $\mathrm{NaCN}$ abundance ratio confirming reaction (1) as the main formation path for $\mathrm{NaCN}$.

Another factor that might affect the estimate of the $\mathrm{NaCN}$ abundance is the effect of the IR pumping on the $\mathrm{NaCN}$ excitation. This effect has been found to be important for other species such as $\mathrm{NaCl}$ (Quintana-Lacaci et al. 2016). However, there is no information available in the literature about the IR ro-vibrational spectrum of $\mathrm{NaCN}$ that might help to estimate the effect of the IR pumping.

\section{Conclusions}

We have obtained interferometric maps with high and intermediate angular resolution of the metal-bearing molecule $\mathrm{NaCN}$. As shown by Guélin et al. (1997) and Petrie (1996), this molecule emission arises in the inner regions of the envelope of IRC +10216 . New maps have shown that this emission presents an inner hole that has previously not been detected. Furthermore, emission from $\mathrm{NaCl}$, as well as its abundance, suggest that when $\mathrm{NaCl}$ declines, $\mathrm{NaCN}$ rises. However, two problems prevent us from confirming reaction (1) as the main formation path of $\mathrm{NaCN}$.

First, the origin of $\mathrm{CN}$ is not clear. Agúndez et al. (2015) did not succeed to model the abundance of this species taking

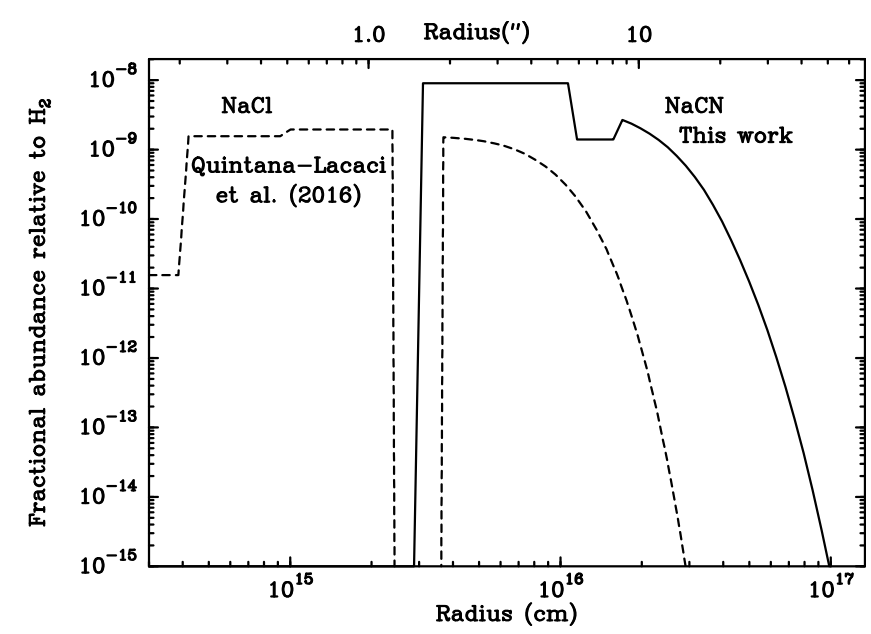

Fig. 5. $\mathrm{NaCN}$ abundance profile obtained assuming LTE conditions (solid line). $\mathrm{NaCl}$ abundance profile obtained by Quintana-Lacaci et al. (2016) (dashed line).

into account the penetration of UV photons into inner layers as a source of $\mathrm{CN}$. A new photochemical model is required to simultaneously explain the source of the $\mathrm{CN}$ and its impact on $\mathrm{CH}_{3} \mathrm{CN}, \mathrm{NaCN}$, and $\mathrm{HC}_{3} \mathrm{~N}$ formation. Obtaining a reaction rate for (1) is essential to solve the competition for $\mathrm{CN}$ for the different chemical paths.

Second, the derived abundance of $\mathrm{NaCN}$ seems to be higher than that of $\mathrm{NaCl}$. This might be a sign of different parent species, or, more probably, an artifact derived from the LTE assumption. Obtaining collisional rates for $\mathrm{NaCN}$ would allow us to solve this problem.

Acknowledgements. The research leading to these results has received funding from the European Research Council under the European Union's Seventh Framework Programme (FP/2007-2013)/ERC Grant Agreement No. 610256 (NANOCOSMOS). We would also like to thank the Spanish MINECO for funding support from grants CSD2009-00038, AYA2012-32032 \& AYA2016-75066C2-1-P. M.A. also ackowledges funding support from the Ramón y Cajal programme of Spanish MINECO (RyC-2014-16277).

\section{References}

Agúndez, M., Cernicharo, J., \& Guélin, M. 2010, ApJ, 724, L133 Agúndez, M., Fonfría, J. P., Cernicharo, J., et al. 2012, A\&A, 543, A48 Agúndez, M., Cernicharo, J., Quintana-Lacaci, G., et al. 2015, ApJ, 814, 143 Agúndez, M., Cernicharo, J., Quintana-Lacaci, G., et al. 2017, A\&A, 601, A4 Cabezas, C., Cernicharo, J., Alonso, J. L., et al. 2013, ApJ, 775, 133

Cernicharo, J. 2012, in EAS Pub. Ser. 58, eds. C. Stehlé, C. Joblin, \& L. d'Hendecourt (Cambridge: Cambridge Univ. Press), 251

Cernicharo, J., Guélin, M., \& Kahane, C. 2000, A\&AS, 142, 181

Cernicharo, J., Daniel, F., Castro-Carrizo, A., et al. 2013, ApJ, 778, L25

Cernicharo, J., Marcelino, N., Agúndez, M., \& Guélin, M. 2015, A\&A, 575, A91 Decin, L., Richards, A. M. S., Neufeld, D., et al. 2015, A\&A, 574, A5

Groenewegen, M. A. T., Barlow, M. J., Blommaert, J. A. D. L., et al. 2012, A\&A, 543, L8

Guélin, M., Lucas, R., \& Cernicharo, J. 1993, A\&A, 280, L19

Guélin, M., Lucas, R., \& Neri, R. 1997, in CO: Twenty-Five Years of MillimetreWave Spectroscopy, eds. W. B. Latter, S. J. E. Radford, P. R. Jewell, J. G. Mangum, \& J. Bally (Dordrecht: Kluwer), IAU Symp., 170, 359

Guélin, M., Patel, N., Bremer, M., et al. 2017, A\&A, in press DOI: 10.1051/0004-6361/201731619

Lucas, R., Guélin, M., Kahane, C., Audinos, P., \& Cernicharo, J. 1995, Ap\&SS, 224, 293

Petrie, S. 1996, MNRAS, 282, 807

Quintana-Lacaci, G., Cernicharo, J., Agúndez, M., et al. 2016, ApJ, 818, 192 Velilla Prieto, L., Cernicharo, J., Quintana-Lacaci, G., et al. 2015, ApJ, 805, L13 


\section{Appendix A: Observations}

The observations were obtained with compact and extended array configurations, with baselines in the range $\sim 12-300 \mathrm{~m}$ and $\sim 30-2000 \mathrm{~m}$, respectively. The field of view (FoV) of the $12 \mathrm{~m}$ ALMA antennas ranges from $\sim 69^{\prime \prime}$ at $84 \mathrm{GHz}$ to $\sim 50^{\prime \prime}$ at $115.5 \mathrm{GHz}$. Additional observations were performed with the IRAM $30 \mathrm{~m}$ telescope to recover the flux filtered out by the interferometer. Observations were centered on the position of the star, with coordinates J2000.0 RA $=09^{\mathrm{h}} 47^{\mathrm{m}} 57.446$ and Dec $=13^{\circ} 16^{\prime}: 43^{\prime \prime} .86$, according to the position of the $\lambda 1 \mathrm{~mm}$ continuum emission peak (Cernicharo et al. 2013). A detailed description of the spectral survey will be presented elsewhere (Cernicharo et al., in prep.). The data were calibrated using the $\mathrm{CASA}^{1}$ software package, and imaged and cleaned with GILDAS $^{2}$ software package. We used the SDI cleaning algorithm since HOGBOM could generate artificially clumpy structures for high spatial resolution observations.

For the emission lines studied here, data from the ALMA compact and extended configurations were merged, after continuum subtraction, with the short-spacing data obtained with the IRAM $30 \mathrm{~m}$ telescope. In particular, since the $\mathrm{NaCN}$ emission is relatively weak, we present low spatial resolution $\mathrm{NaCN}$ maps with high $\mathrm{S} / \mathrm{N}$, obtained by merging the ALMA compact configuration and short-spacing data, and high spatial resolution maps with low S/N, obtained when merging both ALMA configurations plus the short-spacing data (see Table 1 for details). In order to increase the $\mathrm{S} / \mathrm{N}$, we stacked the $\mathrm{NaCN}$ emission of the different lines lying at $3 \mathrm{~mm}$. These maps are presented in Figs. A.1 and A.2.

We also present a map of $\mathrm{MgNC} 8_{17 / 2}-7_{15 / 2}$, observed at the same time as the $3 \mathrm{~mm}$ ALMA line survey. As for $\mathrm{NaCN}$ emission map, we merged single-dish OTF data with those of the extended and compact ALMA configurations. This map is presented in Fig. A.3

In addition, we recently obtained ALMA Cycle-4 data that cover two $\mathrm{NaCN}$ transitions at $\lambda 2 \mathrm{~mm}$ (see Table 1). These observations consisted in a mosaic of seven fields covering a FOV of $\sim 214^{\prime \prime}$. We note that for these transitions we did not have short-spacing data, and thus the maps only rely on ALMA visibilities. Although the $\mathrm{NaCN}$ emission is relatively compact, some flux is expected to be filtered out. The baselines of the interferometer were in the range $\sim 12-408 \mathrm{~m}$. For a detailed description of these ALMA-Cycle 4 observations see Velilla Prieto et al. (in prep.). These maps are shown in Figs. A.4 and A.5.

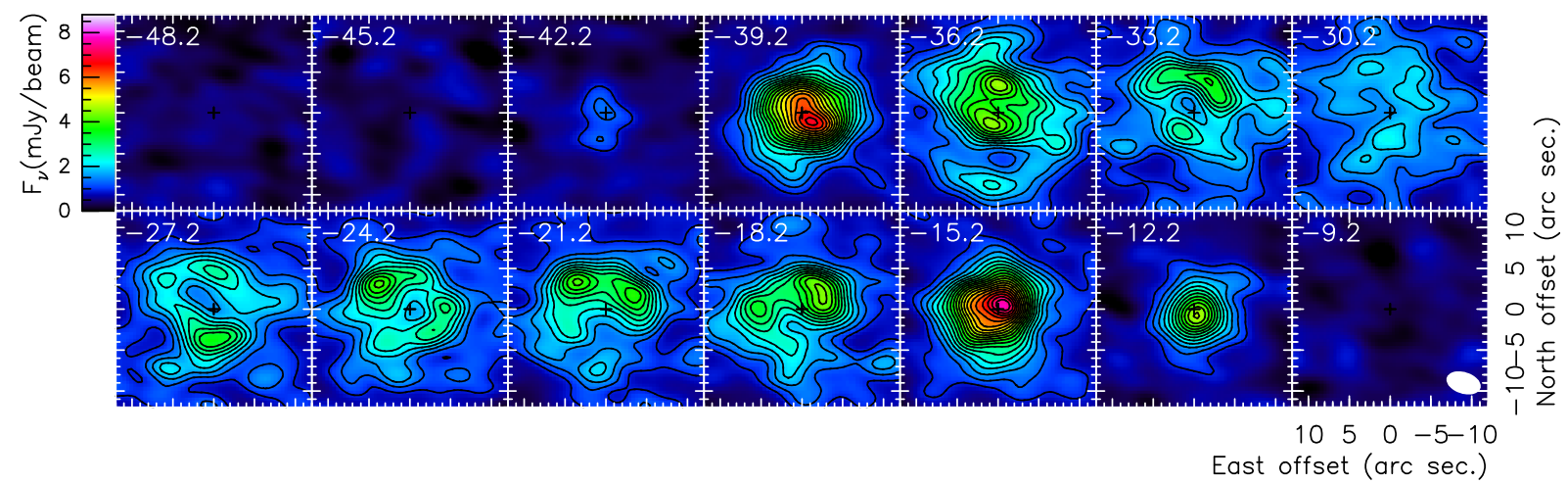

Fig. A.1. Low spatial resolution interferometric map of the $\mathrm{NaCN}$ stacked emission of the transitions at $\lambda 3 \mathrm{~mm}$ presented in Table 1 . In the upper left corner of each panel we note the $v_{\mathrm{LSR}}$ of the channel $\left(V_{\mathrm{sys}}=-26.5 \mathrm{~km} \mathrm{~s}^{-1}\right)$. The lowest contour corresponds to a value of $3 \sigma$, and the rest of the contours are equally spaced in jumps of $2 \sigma$ with respect to the first contour. The rms of the map is $\sigma=0.8 \mathrm{mJy}^{2}$ beam ${ }^{-1}$. The beam size is drawn in the last panel. The HPBW is $4 .{ }^{\prime \prime} 3 \times 2$.' $^{\prime} 6$ with a PA of $72^{\circ}$.

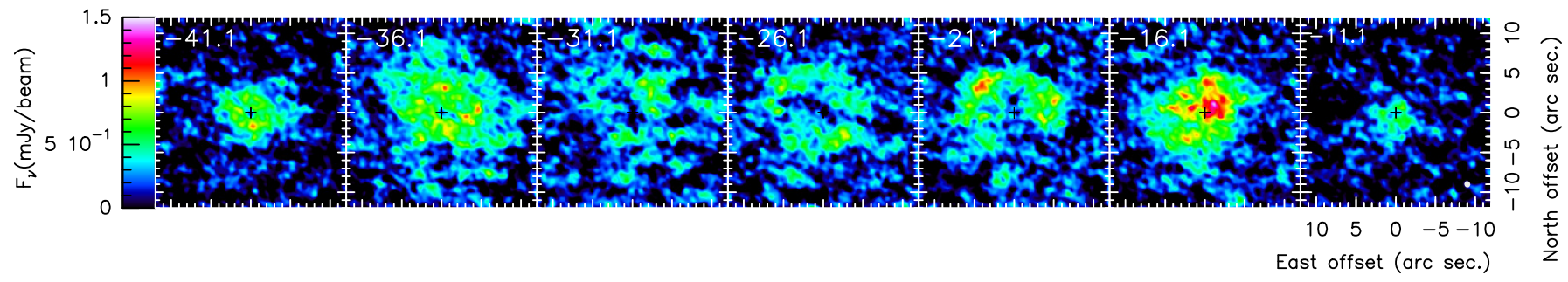

Fig. A.2. High spatial resolution interferometric map of the $\mathrm{NaCN}$ stacked emission of the transitions presented in Table 1. In the upper left corner of each panel we note the $v_{\mathrm{LSR}}$ of the channel $\left(V_{\mathrm{sys}}=-26.5 \mathrm{~km} \mathrm{~s}^{-1}\right)$. The rms of the map is $\sigma=0.76 \mathrm{mJy}_{\mathrm{beam}}{ }^{-1}$. The beam size is drawn in the last panel. The HPBW is $0 .{ }^{\prime \prime} 8 \times 0.0^{\prime \prime} 7$ with a PA of $20^{\circ}$. The flux density scale is in Jy beam ${ }^{-1}$.

\footnotetext{
http://casa.nrao.edu/

2 http://www.iram. fr/IRAMFR/GILDAS/
} 


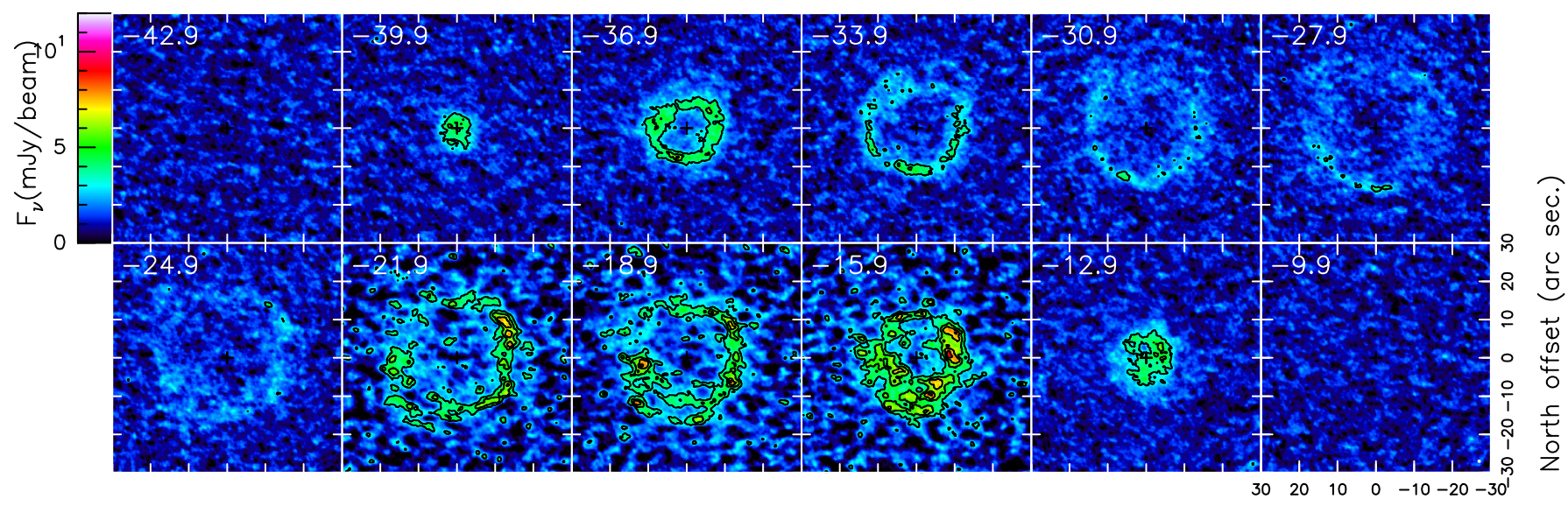

East offset (arc sec.)

Fig. A.3. Interferometric map of the MgNC $8_{17 / 2}-7_{15 / 2}$ emission of the transitions observed within ALMA project 2013.1.00432.S. In the upper left corner of each panel we note the $v_{\mathrm{LSR}}$ of the channel $\left(V_{\mathrm{sys}}=-26.5 \mathrm{~km} \mathrm{~s}^{-1}\right)$. The lowest contour corresponds to a value of $3 \sigma$, and the rest of contours are equally spaced in jumps of $2 \sigma$ with respect to the first contour. The rms of the map is $\sigma=0.9 \mathrm{mJy} \mathrm{beam}^{-1}$. The beam size is downgraded to that of Fig. A. 1 and is drawn in the last panel. The HPBW is $0{ }^{\prime \prime} 9 \times 00^{\prime \prime} 8$ with a PA of $33^{\circ}$. The flux density scale is in mJy beam ${ }^{-1}$.

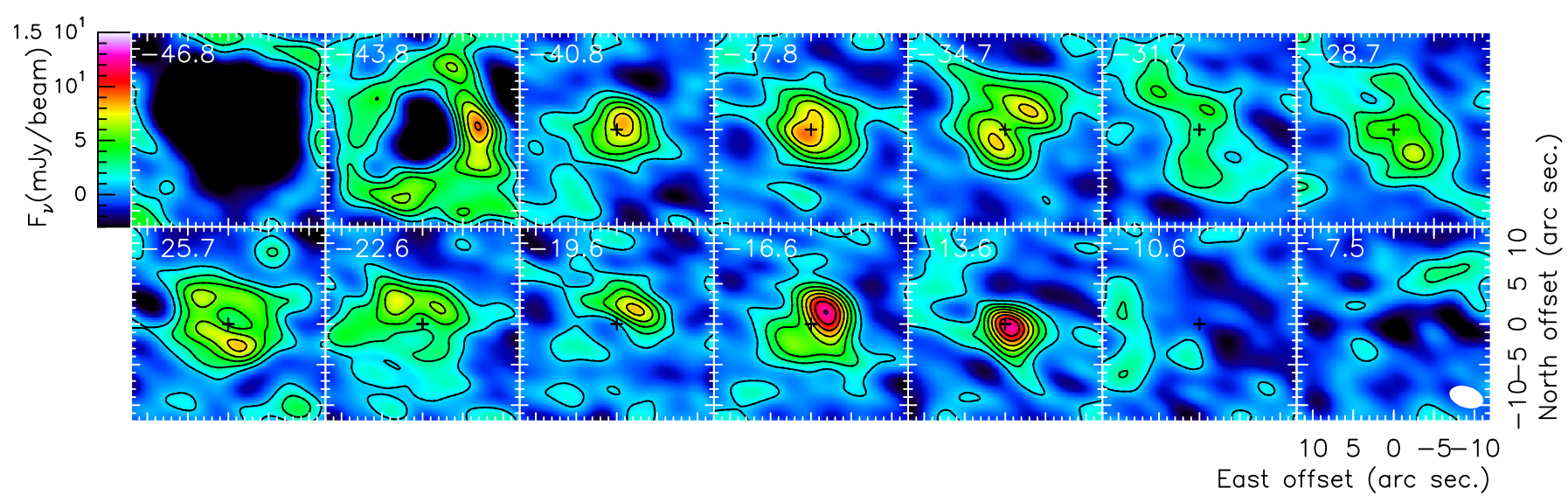

Fig. A.4. $\lambda 2 \mathrm{~mm}$ interferometric map of the $\mathrm{NaCN} 9_{1,8}-8_{1,7}$ emission. The emission of the line $\mathrm{C}_{3} \mathrm{H}_{2} 3_{1,2}-2_{2,1}$ at $145089.61104 \mathrm{MHz}$ is partially blended with that of $\mathrm{NaCN}$ and can be seen in the first channels. The central channels of the $\mathrm{NaCN}$ are, however, free of this pollution. In the upper left corner of each panel we note the $v_{\mathrm{LSR}}$ of the channel $\left(V_{\mathrm{sys}}=-26.5 \mathrm{~km} \mathrm{~s}^{-1}\right)$. The lowest contour corresponds to a value of $1 \sigma$, and the rest of contours are equally spaced in jumps of $1.5 \sigma$ with respect to the first contour. The rms of the map is $\sigma=1.1 \mathrm{mJy} \mathrm{beam}^{-1}$. The beam size is downgraded to that of Fig. A. 1 and is drawn in the last panel. The HPBW is $0 .{ }^{\prime \prime} 8 \times 0 \prime^{\prime \prime} 7$ with a PA of $20^{\circ}$. The flux density scale is in Jy beam ${ }^{-1}$.

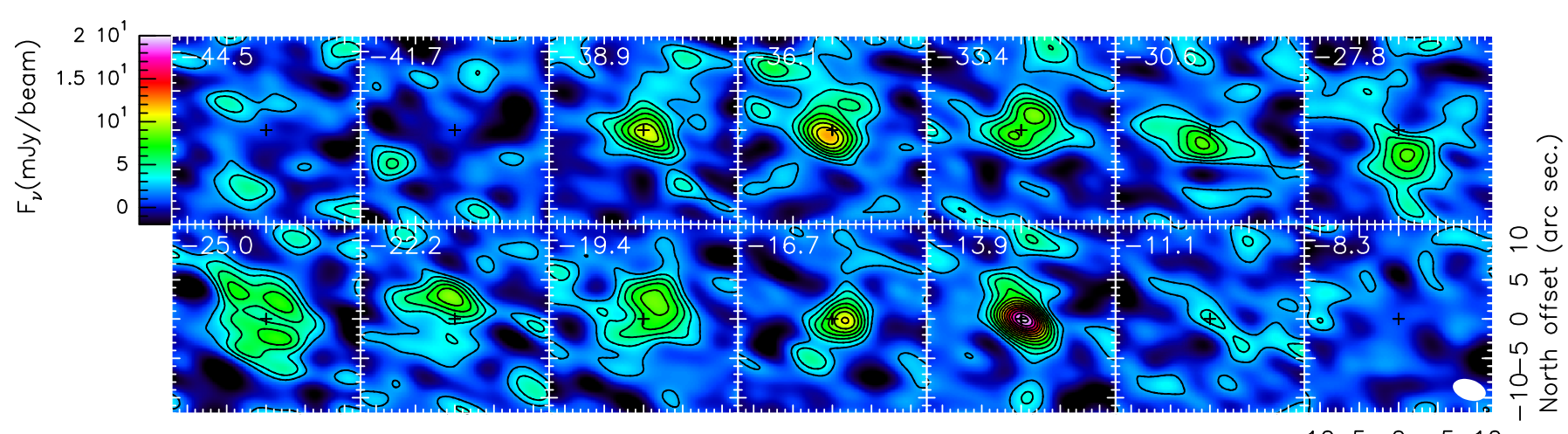

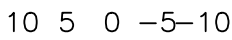

East offset (arc sec.)

Fig. A.5. $\lambda 2 \mathrm{~mm}$ interferometric map of the $\mathrm{NaCN} 10_{2,8}-9_{2,7}$ emission of the transitions presented in Table 1 . In the upper left corner of each panel we note the $v_{\mathrm{LSR}}$ of the channel $\left(V_{\text {sys }}=-26.5 \mathrm{~km} \mathrm{~s}^{-1}\right)$. The lowest contour corresponds to a value of $1 \sigma$ and the rest of contours are equally spaced in jumps of $1 \sigma$ with respect to the first contour. The rms of the map is $\sigma=1.5 \mathrm{mJy}$ beam ${ }^{-1}$. The beam size is downgraded to that of Fig. A.1 and is drawn in the last panel. The HPBW is $0 . \prime 8 \times 0 . \prime 7$ with a PA of $20^{\circ}$. The flux density scale is in Jy beam ${ }^{-1}$. 